\title{
Arzt als Arbeitgeber: Neue Sozialversicherungsgrenzen beachten
}

- Mit den neuen Rechengrößen in der Sozialversicherung werden die für das Versicherungsrecht sowie für das Beitrags- und Leistungsrecht in der Sozialversicherung maßgebenden Grenzen bestimmt. Für das Jahr 2011 gelten folgende Größen: Arbeitnehmer sind nicht gesetzlich krankenversicherungspflichtig, wenn sie im Jahr mehr als 49500 bzw. im Monat mehr als 4125 Euro verdienen. Die Krankenund Pflegeversicherungsbeiträge werden von jährlich höchstens 44550 bzw. von monatlich höchstens 3712,50 Euro berechnet. Die Beitragsbemessungsgrenze für die Renten- und Arbeitslosenversicherung beträgt 66000 Euro in den alten Bundesländern (aBL) bzw. 57600 Euro in den neuen Bundesländern (nBL) im Jahr. Die Renten- und Arbeitslosenversicherungsbeiträge werden von höchstens
5500 Euro (aBL) bzw. 4800 Euro (nBL) monatlich berechnet. Die Bezugsgröße in der Sozialversicherung beträgt 2555 (aBL) bzw. 2240 Euro (nBL) monatlich. Die Geringfügigkeitsgrenze ist bei 400 Euro monatlich geblieben. Der Beitragssatz für die Krankenversicherung erhöht sich für das ganze Bundesgebiet auf $15,5 \%$. Davon tragen Arbeitgeber 7,3\% und Arbeitnehmer 8,2\%. Der Beitragssatz für die Pflegeversicherung beträgt 1,95\% bzw. bei Kinderlosen, die das 23. Lebensjahr bereits vollendet haben, 2,2\%, der Rentenversicherungsbeitragssatz 19,9\%. Der Beitragssatz für die Arbeitslosenversicherung steigt auf 3\%.

\section{MMW Kommentar}

Beiträge zur Kranken-, Pflege-, Rentenund Arbeitslosenversicherung müssen in der Regel je zur Hälfte vom Arbeitgeber getragen werden. Bei der Krankenversi-

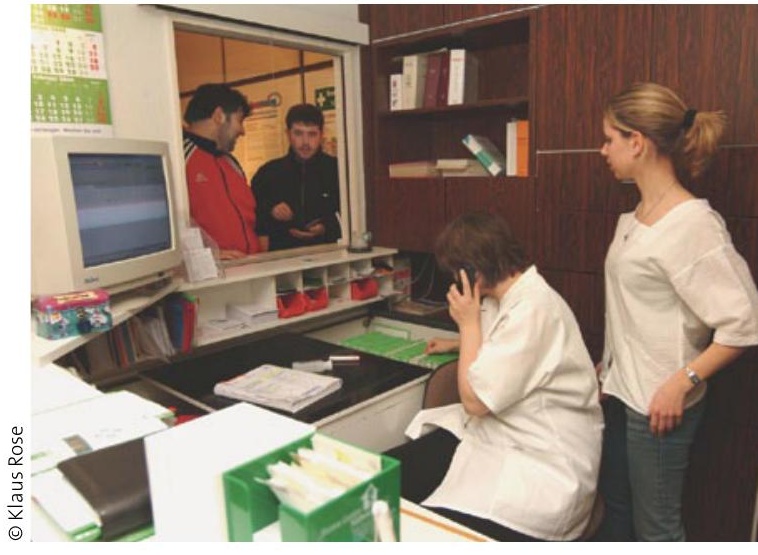

„Praxisperlen“ sind teurer geworden.

cherung muss der Arbeitnehmer allerdings zusätzlich einen Anstieg um 0,9\% selbst tragen. Auch der Beitragszuschlag zur Pflegeversicherung für Kinderlose von $0,25 \%$ ist vom Arbeitnehmer allein zu tragen. Ausnahmen gelten für das Bundesland Sachsen. Dort trägt der Arbeitnehmer 1,475\% bzw. der kinderlose Arbeitnehmer nach Vollendung des 23. Lebensjahres 1,725\% und der Arbeitgeber 0,475\% des Beitrags zur Pflegeversicherung.

\section{Deutschland bei gesundheitlichen Risikofaktoren Spitze}

- Die Europäische Kommission hat gemeinsam mit der OECD im Dezember 2010 den Bericht „Gesundheit auf einen Blick: Europa 2010“ herausgegeben, der die Gesundheitssysteme und Gesundheitsausgaben in der EU-27 und den drei EFTA-Ländern (Island, Norwegen und Schweiz) darstellt und Trends zum Thema Gesundheit aufzeigt.

Im Besonderen macht der Bericht auf den Anstieg von Übergewicht und Fettleibigkeit aufmerksam. So seien im Durchschnitt über 15\% der Erwachsenen in der EU von Fettleibigkeit betroffen. In Deutschland tritt laut dem „Integrierten Forschungs- und Behandlungszentrum Adipositaserkran-

kungen“ insgesamt bei 47,8\% der Erwachsenen ein Übergewicht auf. $14,8 \%$ der Kinder und Jugendlichen zwischen 2 und 17 Jahren gelten als übergewichtig und $6,1 \%$ davon seien

\section{MMW Kommentar}

Angesichts einer solchen Entwicklung stellt sich die Frage nach Sinn und Zweck einer morbiditätsbedingten Vergütung der Ärzte und deren Verfeinerung durch die (Zwangs-)Einführung von Kodierrichtlinien. Die Verfechter dieser Maßnahme sind der Auffassung, dass bei dieser Verknüpfung das ärztliche Honorar steigen wird. Die Grundüberlegung ist dabei richtig, die Folgen aber nicht finanzierbar. Zu fettleibig. Insgesamt leben in Deutschland 1,7 Millionen übergewichtige Kinder, etwa 750000 davon seien fettleibig.

diesem Ergebnis werden die politisch Verantwortlichen in unserem Land zwangsläufig kommen, wenn es darum gehen wird, den Morbiditätsbezug als Grundlage des Arzthonorars dauerhaft einzuführen. Das kann zwar ab 2013 der Fall sein, man muss aber schon ein Superoptimist sein, wenn man an eine solche Entwicklung angesichts dieser Daten zur Entwicklung der Volksgesundheit glaubt. 